\title{
DOSINDYGO - DOSe finding for INtraoperative photoDYnamic therapy of GliOblastoma
}

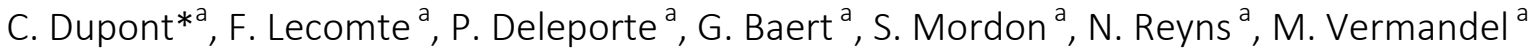 \\ aUniv. Lille, Inserm, CHU Lille, U1189 - ONCO-THAI - Image Assisted Laser Therapy for Oncology, F- \\ 59000 Lille, France
}

\begin{abstract}
Glioblastoma is a malignant brain tumor with a poor prognosis. Currently, complete resection is rarely feasible, since tumor cells usually infiltrate the surrounding brain. Recently, the INDYGO clinical trial has been achieved to assess the toxicity of photodynamic therapy (PDT) delivered intraoperatively to treat newly diagnosed glioblastoma. Today, we believe that the PDT effect obtained in the INDYGO clinical trial can be improved by a higher light dose.

The DOSINDYGO clinical trial aims to achieve a light-dose escalation increasing up to four times the initial light dose used in the INDYGO trial. An increase of both light power and treatment time should allow to treat deeper in the surrounding tissues (up to $8 \mathrm{~mm}$ ) and thus decrease the recurrence risk. First light dose will be reached by doubling the treatment time used in the INDYGO trial, the other one will be achieved by increasing light power only. This methodology was chosen in order to maintain an acceptable treatment time for anesthesia but also to prevent higher fluence rate that could induce a lower tolerance as observed in our preclinical results.

Primary endpoint will be to determine the optimum light-dose regarding the ratio efficacy and tolerance of the treatment. Primary criterion is the assessment of the progression free survival within the bed border's cavity.

Finally, although no adverse effect has been noticed during the INDYGO trial, increasing light dose in this DOSINDYGO trial could result in other direct and indirect biological effects.
\end{abstract}

Keywords: photodynamic therapy, glioblastoma, clinical trial, light dose escalation 


\section{INTRODUCTION}

Glioblastoma (GBM) is a malignant brain tumor with a particularly poor prognosis, with a median overall survival of approximately 15 months with the current standard of care (SOC) [1-3]. Although GBM is a rare neoplastic disease with low prevalence $(0.3 / 10,000$ persons), it remains the most frequent primary malignant brain tumor in adults $[4,5]$. Currently, no existing therapeutic agent is able to stop GBM progression, and complete resection is rarely feasible, since tumor cells usually infiltrate the surrounding brain: adjuvant therapies to improve local control are thus highly expected.

Nowadays, European Society of Medical Oncology (ESMO) guidelines provide recommended treatment options according to the grade of the glioma [6]. Given the invasive nature of GBM, local relapse is a universal occurrence, a reason why treatment is usually completed by radiotherapy (RT) to the tumor bed, and concomitant and adjuvant chemotherapy (TMZ), which prolongs Progression Free Survival (PFS) and Overall Survival (OS). Although adjuvant treatment after surgery significantly improves results, the benefits remain modest (less than 3 months of increase in the median OS with RT+TMZ versus RT alone [3]). Therefore, management of newly diagnosed patients should include maximal resection when achievable. The Extent Of Resection (EOR) is of prognostic value.

Recently, Fluorescence Guided Resection (FGR) of GBM has been developed using 5-aminolevulinic acid (5-ALA). An increase of EOR and an improvement in PFS has been observed when 5-ALA is used for FGR [7]. However, it has been shown that extended resections performed using FGR carries the risk of temporary impairment of neurological function [8]. Furthermore, relapse occurs in the cavity margin in $85 \%$ of cases [9], and the improvement of local control by EOR and additional therapy delivered intraoperatively [10] is a key point of success in GBM management. In this context, 5-ALA PhotoDynamic Therapy (PDT) appears relevant to treat areas bordering the resection cavity while maintaining a maximal but safe resection.

PDT is a non-thermal energy based therapy relying on light exposure after drug accumulation in the tumor cells. A PDT effect is the result of the synergy of three elements: a drug named photosensitizer (PS), the energy delivered through light exposure at a specific wavelength to excite the PS and the presence of oxygen. Combination of these three components leads to the formation of reactive species of oxygen, including singlet oxygen, which are cytotoxic molecules that damage the PS-targeted cells.

In the context of GBM treatment, 5-ALA, which is already administered for FGR purpose, is a prodrug that is converted into protoporphyrin IX (PpIX), as part of the endogenous heme synthesis. PpIX is a photoactive compound that absorbs light at specific wavelengths and that is the PS specifically accumulated in tumor cells. 
By taking advantage of the presence of 5-ALA for FGR purpose in remaining tumor cells, we propose to apply 5-ALA-mediated PDT early after surgery in order to improve the local control by treating infiltrating and non-resected GBM cells.

\section{INDYGO: A FEASIBILITY STUDY}

The concept of our therapeutic approach is to deliver 5-ALA PDT intraoperatively early after FGR with a simple approach suitable to be included in the surgical workflow. A specific light applicator $[11,12]$ has been designed to safely deliver light at the required wavelength into the surgical cavity. This medical device is composed of two main parts. The first one is a light applicator composed of a single-use inflatable balloon and a reusable trocar. This light applicator delivers the laser light inside the cavity through the help of an optical fiber placed in the middle of the balloon. Once the balloon placed inside the cavity, it is inflated by injection of a white liquid used to homogenize the light distribution around the balloon. Thus, all tissues surrounding the balloon are illuminated homogeneously during the treatment. The second part is a laser device dedicated to the treatment delivery. By knowing the quantity of liquid injected in the balloon, the device computes automatically the lighting duration in order to deliver a light dose of $25 \mathrm{~J} / \mathrm{cm}^{2}$ at $5 \mathrm{~mm}$ depth inside brain tissues. This light dose is considered as the limit where we can expect to obtain a therapeutic effect.

This device has been evaluated in the first feasibility study on intraoperative 5-ALA PDT for newly diagnosed glioblastoma named INDYGO [13]. This trial was leaded by Pr. Nicolas Reyns from Neurosurgery Department of the University Hospital of Lille, France. The Primary endpoint was the feasibility of intraoperative photodynamic therapy early after surgical resection of glioblastoma without unacceptable and unexpected toxicities. Ten patients have been recruited and follow-up phase is still ongoing.

Alongside performance of the PDT trial, biomarkers have been investigated. The main objective concerning biomarkers within this application was to obtain new data on the effectiveness of the PDT by monitoring Multiparametrics Magnetic Resonance Imaging (MRI). Additionally, biological samples have been collected during the trial to enable future exploratory studies on the evolution of immune cell subpopulations, the secreted factors and the nanovesicules released into the blood. 


\section{DOSINDYGO: A LIGHT DOSE ESCALATION CLINICAL TRIAL}

Today, we believe that the PDT effect obtained in the INDYGO clinical trial can be improved by a higher light dose. Indeed, an increase of both power light and treatment time should allow to treat deeper in the surrounding tissues (up to $8 \mathrm{~mm}$ ) and thus decrease the recurrence risk. It appears essential to find the optimum light dose to improve the patient's survival without the toxicity of the therapy.

The DOSINDYGO clinical trial aims to achieve a light-dose escalation increasing up to four times the initial light dose used in the INDYGO trial.

Primary endpoint will be to determine the optimum light-dose regarding the ratio efficacy and tolerance of the treatment. Primary criterion is the assessment of the PFS within the $25 \mathrm{~mm}$ in bed border's cavity associated to the evaluation of toxicities every 3 months until relapse.

Secondary endpoints are the evaluation of the PFS and OS determined from the date of diagnosis of glioblastoma as defined by international standards (Revised Assessment in Neuro-Oncology -RANOcriteria), response to treatment assessed by MRI exams every 3 months after intraoperative PDT procedure until recurrence, quality of life assessed by questionnaires (QLQ-C3O and QLQ-BN20) preand post-PDT procedure every 3 months until recurrence.

Primary, newly diagnosed, non-recurrent and resectable GBM will be recruited. In the first stage of the light-dose escalation, 3 patients will be included. Then, an independent surveillance comity will review the potential toxicity encountered during this first stage. Under the approval of the committee, 3 others patients will be recruited for the second light-dose stage. Under a second approval of the committee, 3 others patients will be recruited for the third light-dose stage. If one toxicity is observed, 3 more patients will be added to the same light-dose stage. If two toxicities appear at the same light-dose, the study will end and the previous light dose will be declared as optimum light-dose.

As proposed in the INDYGO trial, an ancillary study will be performed to assess potential biomarkers of a PDT treatment immune response. Recent preclinical studies have shown a growing interest in a PDT impact on immune system [14, 15]. PDT involves innate and acquired specific immunity, as dying cells express markers recognized by dendritic cells. These cells can activate T lymphocytes specifically directed against tumor cells. Thus, a temporary "boost" of immune system seems to be induce early after PDT stimulation. We plan to carry on clinical investigations begin in the INDYGO trial to demonstrate the immune PDT impact.

This study will be leaded by the Pr. Nicolas Reyns from the Neurosurgery Department of the University Hospital of Lille, France declared as study sponsor. Two recruitment centers will be soliciting: neurosurgery departments of the University Hospital of Lille, France and Erasme Hospital. 
Due to the large population treated by those two reference centers (about 150 and 50 GBM diagnosed each year in the neurosurgery Department of the University Hospital of Lille and Erasme Hospital respectively), about one year will be required to complete the recruitment of all patients.

In the medium term, the objective is to ensure the non-toxicity of the procedure in order to propose the PDT with the optimal dose of light. The PDT thus optimized would be the subject of a phase III clinical study for the PDT treatment of GBM. In the long term, the aim of the project is to include the PDT in the GBM SOC.

\section{REFERENCES}

[1] G. lacob, and E. B. Dinca, "Current data and strategy in glioblastoma multiforme," J Med Life, 2(4), 386-93 (2009).

[2] Q. T. Ostrom, L. Bauchet, F. G. Davis et al., "The epidemiology of glioma in adults: a "state of the science" review," Neuro Oncol, 16(7), 896-913 (2014).

[3] R. Stupp, W. P. Mason, M. J. van den Bent et al., "Radiotherapy plus concomitant and adjuvant temozolomide for glioblastoma," N Engl J Med, 352(10), 987-96 (2005).

[4] J. Grill, [Glioblastoma], (2007).

[5] J. L. Fisher, J. A. Schwartzbaum, M. Wrensch et al., "Epidemiology of brain tumors," Neurol Clin, 25(4), 867-90, vii (2007).

[6] R. Stupp, M. Brada, M. J. van den Bent et al., "High-grade glioma: ESMO Clinical Practice Guidelines for diagnosis, treatment and follow-up," Ann Oncol, 25 Suppl 3, iii93-101 (2014).

[7] W. Stummer, U. Pichlmeier, T. Meinel et al., "Fluorescence-guided surgery with 5aminolevulinic acid for resection of malignant glioma: a randomised controlled multicentre phase III trial," The lancet oncology, 7(5), 392-401 (2006).

[8] W. Stummer, J. C. Tonn, H. M. Mehdorn et al., "Counterbalancing risks and gains from extended resections in malignant glioma surgery: a supplemental analysis from the randomized 5-aminolevulinic acid glioma resection study. Clinical article," J Neurosurg, 114(3), 613-23 (2011).

[9] K. Petrecca, M. C. Guiot, V. Panet-Raymond et al., "Failure pattern following complete resection plus radiotherapy and temozolomide is at the resection margin in patients with glioblastoma," J Neurooncol, 111(1), 19-23 (2013). 
[10] V. Pavlov, P. Page, G. Abi-Lahoud et al., "Combining intraoperative carmustine wafers and Stupp regimen in multimodal first-line treatment of primary glioblastomas," Br J Neurosurg, 29(4), 524-31 (2015).

[11] M. Vermandel, S. Mordon, C. Dupont et al., [SYSTEM FOR TREATMENT BY PHOTODYNAMIC THERAPY OF A CAVITY OF A PATIENT'S BODY AND METHOD FOR PREPARATION OF SUCH SYSTEM], European Patent Office, EP16305347.3 (2016).

[12] C. Dupont, S. Mordon, P. Deleporte et al., "A novel device for intraoperative photodynamic therapy dedicated to glioblastoma treatment," Future Oncology, 13(27), 2441-2454 (2017).

[13] C. Dupont, M. Vermandel, H. A. Leroy et al., "INtraoperative photoDYnamic Therapy for GliOblastomas: Study Protocol for a Phase I Clinical Trial," Neurosurgery, nyy324-nyy324 (2018).

[14] H. Hirschberg, K. Berg, and Q. Peng, "Photodynamic therapy mediated immune therapy of brain tumors," Neuroimmunology and Neuroinflammation, 5(7), 27 (2018).

[15] M. Wachowska, A. Muchowicz, and U. Demkow, "Immunological aspects of antitumor photodynamic therapy outcome," Central-European Journal of Immunology, 40(4), 481-485 (2015). 